\title{
Cultural Sensitivity of a Community-Based Falls Prevention Program Targeting Older Arab American
}

\author{
Lan Yao*, Suha A. Kridli \\ School of Nursing, Oakland University, Rochester, MI, USA \\ Email: *lyao@oakland.edu
}

How to cite this paper: Yao, L. and Kridli, S.A. (2018) Cultural Sensitivity of a Community-Based Falls Prevention Program Targeting Older Arab American. Open Journal of Nursing, 8, 835-847. https://doi.org/10.4236/ojn.2018.811063

Received: October 31, 2018

Accepted: November 25, 2018

Published: November 28, 2018

Copyright (๑) 2018 by authors and Scientific Research Publishing Inc. This work is licensed under the Creative Commons Attribution International License (CC BY 4.0).

http://creativecommons.org/licenses/by/4.0/

\section{(c) (i) Open Access}

\begin{abstract}
Falls are a frequent and costly cause of injuries and functional decline in the elderly. Tai Chi is a cost-effective strategy for preventing falls in older adults. Many senior centers have introduced Tai Chi programs to increase mobility and decrease the risk of falls. However, the practice has yet to be widely disseminated to ethnic minorities who are not culturally connected to Tai Chi. This paper describes implementation barriers and recruitment and retention challenges of Arab American participants in a Tai Chi intervention-based health promotion program, including issues related to community organization and staffing, recruitment and retention, need for building relationships, need for translation and interpreters, and cultural barriers \& misconceptions. Understanding and paying adequate attention to these challenges may help facilitate in planning other health promotion interventions targeting Arab American population.
\end{abstract}

\section{Keywords}

Elderly Care, Falls, Arabic, Culturally Sensitive, Prevention, Community-Based Intervention Program

\section{Introduction}

More than 3.6 million Arab-Americans reside in the United States [1]. Metropolitan Detroit has the largest concentration of Arabs in the world outside of the Middle East (California's Arab population is more spread out) [1] while Arab-Americans are the third largest ethnic population in the state of Michigan. Arab Americans have a strong sense of cultural and historical identity and pride, and most speak Arabic. 
An Arab refers to an individual who was born in an Arab country, speaks Arabic language, and shares the values and beliefs of an Arab culture [2]. Arab Americans can be defined as persons who trace their ancestry to the northern African countries of Morocco, Tunisia, Algeria, Libya, Sudan, and Egypt, as well as the western Asian countries of Iraq, Iran, Lebanon, occupied Palestine, Syria, Jordan, Bahrain, Qatar, Oman, Saudi Arabia, Kuwait, United Arab Emirates, and Yemen. Based on the Arab American Institute report [1], the majority of Arab Americans are native-born, and nearly $82 \%$ of Arabs in the U.S. are citizens. Aging related health problems become increasingly challenging to older Arab Americans. Arab Americans have higher age-adjusted mortality risk particularly due to chronic diseases, compared with non-Arab and non-Hispanic Whites [3]. Limited preventive health practices are major concerns for Arab community advocates. "Most Arab seniors are active physically and socially in their home countries, but not in the United States" due to lack of opportunities and access including language barriers

(http://www.arabamerica.com/creating-the-right-settings-for-arab-american-sen iors/).

Each year, one in every three adults ages 65 or older fall and 2 million are treated in emergency departments for fall-related injuries (The Centers for Disease Control and Prevention). Direct medical costs (patients and insurance company's payment) for falls totaled $\$ 34$ billion. Near $90 \%$ hospitalizations after an elderly fall were due to simple falls and the fallers' short-term mortality was $6 \%$ [4]. Many elders fear of another fall thus results in loss of confidence and self-imposed restriction of activities. Those who develop either falls or fear of falling are at risk for developing the other, with a resulting spiraling risk of falls, fear of falling, and functional decline [5]. Falls also contributes to caregiver burden, impedes the caregiver's ability to undertake activities of daily living, and increases caregiver stress [6] [7]. As the state of Michigan experiences considerable growth in its older population including older Arab Americans, the rising number of falls and the costs to manage fall injuries underscore the need for dissemination and implementation of evidence-based fall prevention interventions.

Falls in older adults are preventable through exercise interventions. Tai Chi is an exercise which emphasizes slow movements that enhance body alignment and improve balance, so are suitable and safe for older adults. Tai Chi movements demand body alignment and stance balance and are usually performed in a semi-squat position to enhance loading of the muscles and motion ranges of the lower limb and joints. Evidence indicates that long- and short-term Tai Chi practice in the older population has additional benefits including [8]: reduced fear of falling; decreased musculoskeletal pain; improved sleep; lowered stress levels; improved emotional health, psychosocial status and immune function; increased self-efficacy; and improved executive and cognitive function. CDC has a complied inventory which contains evidence-based fall prevention interventions that can be adopted for use in community settings. Among these, Tai Chi is 
an especially effective exercise approach in improving strength and functional balance, thus reducing fear of falling and the risk of falls in older adults including those with balance problems [9] [10]. Tai Chi is a proven evidence-based fall prevention exercise intervention that can be used by public health and clinical practice communities to reduce the risk and incidence of falls and that have the potential for a positive return on investment [9] [10]. Although there is an increasing effort to disseminate Tai Chi program into community practice, there remains a significant gap in translating and disseminating such program to community settings that involve older Arab Americans.

This paper describes an adapted Tai Chi exercise program, program implementation barriers, and recruitment and retention challenges of Arab American participants. Information reported are not intended to be generalized to all Arab American communities due to it is a program evaluation at one community center. The information is derived from the authors' experience in conducting the program, which aims to reduce falls in older Arab-Americans.

\section{Method/Program Description}

In collaboration with an Arab community center in Dearborn, MI, a Tai Chi for Falls program was introduced to older Arab Americans who sought services at the center. As older adults become less physically active and more socially isolated to avoid situations that could lead to falls [11], early interventions are important. Thus our program targeted older adults who are 55 years and older. The inclusion criteria included: 1) $\geq 55$ years, 2) self-identified Arab American, understand Arabic and/or English, 3) Ambulatory with or without assistive devices, 4) Vision and hearing adequate to support usual communication, and 5) Medically stable, no contraindications to standing physical exercise (e.g., severe lumbar spine, hip, or knee arthritis that limits exercise capability).

Direct and indirect recruitment methods were used. Indirect recruitment involved posting bilingual flyers and brochures through the community center and distributing them to community center contacts. Direct recruitment efforts involved the community center bilingual staff coordinator talking to prospective participants in person at the center and following up on the conversation via phone. Older adults who were interested in the program signed up with the center. After explaining the intervention program in Arabic and English and had the individuals consented, the center staff coordinator scheduled an on-site screening and health assessment appointment with a $\mathrm{RN} /$ project director and a bilingual research assistant at the community center to determine that they met the recruitment criteria and it was safe for them to participate. This program was reviewed and approved by the University Institutional Review Board (IRB).

The Tai Chi sessions were provided separately for different genders (i.e., male sessions on Tuesday and Thursday and female sessions on Wednesday and Friday). Twice-weekly 1-hour classes led by an experienced Tai Chi instructor were provided in a classroom for 12 weeks at the community center. Based on our previous experience working with Arab-Americans and suggestions from com- 
munity center staff, a female Tai Chi instructor was culturally acceptable to lead both male and female groups. All classes began and ended with a 5 minutes warm-up and 5 minute of cool-down adapted Tai Chi movements, which emphasize breathing and relaxation. The Tai Chi practice period was chosen per recommended exercise dose based on clinical guidelines [12] and minimum dose to achieve health benefits in implementation studies [9]. As per our previous program, it included six main exercise moves selected on the basis of Yang-style Tai Chi forms which focus on weight shifts in multiple directions and unipedal stance [13]. Participants were instructed to complete the moves one after another slowly in a workout routine, together with others in a group. Instructions of exercise moves were provided in Arabic and English. A trained community center staff coordinator attended each session and translated the instructor's instructions to Arabic.

Assessments were administered by a trained bilingual research assistant, to make sure all instructions were fully understood and measurement protocols were followed. Falls efficacy were assessed by Falls Efficacy Scale-International (FES-I) [14], one of the best existing measures of fear of falling [15]. The FES-I has been used among elderly subjects in diverse populations and been described as a reliable and valid measure of fear of falling among elderly persons in different countries [14] [15] [16]. The Arabic version of the FES-I [16] was available for Arabic-speaking elderly persons when needed. FES-I Scores ranged from 16 to 64 with higher scores indicating higher levels of managing risk of falls.

Participants' functional mobility performances were also conducted. Timed Up and Go test (TUG), a timed sequence of rising from a chair, walking 3 meters, turning, and returning to the chair [17], assesses a person's mobility and requires both static and dynamic balance. A score $>14$ seconds is a reliable and simple indicator for fall risk [18] [19]. Other robust tests of balance and gait [20] [21] [22] were also used including Unipedal Stance Time (UST), a one-legged stance duration time and Gait Speed. Finally, number of falls was counted as total incidence of falls using an exercise log. A fall was defined as an event in which a participant unintentionally came to rest on the ground or floor [23].

\section{Result/Program Implementation Challenges}

The challenges we experienced are organized into community organization \& staffing, recruitment and retention barriers, need for building relationships, need for translation and interpreters, and cultural barriers \& misconceptions. These challenges shared part of the common barriers to minority groups but also possessed distinctive barriers to the Arab Americans.

\subsection{Community Organization \& Staffing}

Partnering with local community organizations is a recognized aspect to successfully implement a fall prevention program [24]. Although this program was developed collaboratively by the research team and the community organization, staff shortage was a big challenge. We had one part-time center staff member 
who worked as the program coordinator. Among other regular responsibilities at the center and things like distributing flyers and brochures, translating and facilitating Tai Chi classes, and serving as a liaison between the university and the site, it was challenging for the staff to put in adequate hours to interact, to encourage and motivate potential participants. In addition, the facility was being renovated and the large meeting room was not available to the team to run large-sized Tai Chi classes of over 10 participants.

It took several attempts to get the attention of the elderly persons, and not all persons to whom the site staff approached were interested in participating. A total of 29 older adults agreed to have their names on the potential participant list, but it was challenging to get appointments with all of them. Resource constraint was one of the barriers for community-based organizations to adopt evidence-based programs, in addition to program adaptation challenges and community culture conflicts [25].

\subsection{Recruitment \& Retention Barriers}

Recruitment remained as the biggest challenge to the program. Recruitment challenges are not rare to health promotion programs [26] [27] [28], where many strategies have been reported. Key shared barriers [27] among different minority and ethnic groups included: 1) mistrust and consequent fear of participation, 2) stigma related to research participation, and 3) competing demands.

Distinct barriers found in this program included males Arab Americans were more reluctant to participate in a health promotion program than their female counterpart. The ratio of male to female volunteers who agreed to be contacted for participation was 1:3. This might have due to the fact that women utilize the health care system more due to childbearing and child-raising. Eighteen females and six males attended baseline testing at the community center as of the current date. Arabic males are traditionally the breadwinner in the house who face more competing demands. What are the driving issues in recruiting male participants is not clear. Our anonymous exit survey with the participants who completed the program $(n=9)$ indicated that none of the participants had practiced Tai Chi before, and seven of them had not heard about what Tai Chi was in the past. A lack of perceived benefits and perceived harm, with and without the prevention program, seemed to be critical issues based on our experience and observation.

To help potential Arab American participants know more about balance enhancement exercises such as Tai Chi, the importance of falls prevention, and the benefits of this program, flyers and brochures did not seem to be an effective way for passing the information. Minority recruitment often require additional investments of time and resources to learn what methods may work in distinct communities to improve community acceptance of clinical research and thus improve participation [27]. Transportation and time commitment remained as barriers. Seven female volunteers did not finish the classes due to lack of time and/or no transportation, although the anonymous exit survey with other participants who completed the program $(n=9)$ were positive. 
Published researches reported that direct (consisting of an introductory lecture with the help of a local contact person in each community center) and culturally specific approach was more productive in recruiting elders from ethnic minority groups, while indirect recruitment through newspaper announcement worked fine in recruiting non-minority whites [26]. Others reported that Hispanics were more responsive to recruitment via family/friends and phone calls, and American Indians were more likely to be reruited from community screening events and medical referrals [28]. Our experience and result supplemented these findings. Mass distribution of brochures/flyers was not effective among Arab Americans. All participants in our program were recruited by the bilingual community center staff coordinator through talking to potential participants in person at community center and followed up with phone conversations.

\subsection{Need for Building Relationships}

Perceptions of trust and mistrust of the program have been found to be a central barrier to recruitment and participation, particularly among minority groups [28]. A common approach to building trust and alleviating attitudinal barriers was community involvement, particularly in the form of using lay outreach workers from the targeted population. Researchers have used peers to successfully recruit and retain minority participants [28]. From our experience, utilizing the community center staff, registry, and network may not have been sufficient. Staying connected with potential participants and building a positive rapport may need to be maintained.

Using staff member whose background is similar to study participants has been reported to significantly improve overall participant numbers and specific subpopulations of research subjects and adding to the success of the study [29]. Our experience was in agreement with this finding. It was found that a senior center staff would have fitted this role better than a young staff, because there is no generation gap between the staff and the participants. The staff member's level of enthusiasm and passion about the health promotion program is also important since she should be the chaperon and motivator for the team.

\subsection{Cultural Barriers \& Misconceptions}

Differences in language, education, and income present unique challenges for interventions that are targeted at healthy behaviors [30]. Arab Americans have a strong sense of cultural and historical identity and pride, and most speak Arabic. Arabic culture doesn't seem to encourage prevention health care. "Wait and see" is many individuals' approach in terms of healthcare. Many Arabic-speaking immigrants would intentionally delay access to medical services even when signs of abnormalities have appeared in terms of blood glucose care [31]. On the contrary, Caucasian English-speaking participants had no reluctance to access and use medical services, their treatment-seeking behaviors were straightforward [31]. This type of illness and treatment perceptions of the Arabs negatively influenced 
their adherence to diabetes self-care activities [32] Misconceptions about physical activity, including that it must be strenuous or uncomfortable to benefit health and a lack of understanding related to how regular, moderate physical activity can benefit health are also problems [30]. Adaptations addressing Arabs' specific health-related habit and beliefs need to be facilitated because they affect Arab Americans engagement in preventive care.

Resnicow's Deep and Surface Structures model [33] [34] may be applied to explain some of the cultural barriers that were identified. Deep structure [33] [34] explains how Arabic cultural, social, psychological, historical, and environmental factors influence health behaviors differently from other racial populations. The deep structure requires understanding of how Arab Americans perceive the cause, course, and treatment of illnesses (e.g., falls) and how their perceptions influence specific healthy lifestyle behaviors (e.g., fatalism, believe that individual health or illnesses are affected by factors beyond own control). The surface structure includes incorporating an appropriate channel of intervention and using people, language, foods, clothing, music, and locations that are familiar to and preferred by Arab Americans [33] [34].

Our program is a female bilingual community center staff delivered program, with a female certified Tai Chi for Health instructor lead the Tai Chi class and train the center staff. We also divided the groups into male and female participant groups to make the program cultural sensitive. Due to limitations of the staff's time commitment, she could not lead the program as was planned. The lesson we learned is that there should always be two staff coordinators from the local site, so that influences associated with staffing challenges and/or commitment could be minimized.

\section{Discussion}

As people age, they often suffer progressive deficits in health and function following a continuum that leads to frailty, disability, and death. Inactivity is problematic for older individuals and particularly prevalent among those with co-morbidities. Tai Chi is a cost-effective strategy for optimizing spending to prevent falls and maximize health gains in people [10] [35]. It is more effective than resistance training or stretching, and had the lowest cost [35]. It can be broadly implemented in community senior centers for primary prevention of falls among community-dwelling older adults [9]. Recent works [36] [37] [38] [39] [40] suggested that Tai Chi has considerable potential for conferring health benefits on low-income, ethnically diverse community dwelling elderly who have complex chronic illnesses, since it is effective yet does not require equipment and offers flexibility in terms of space needs [36] [37] [38] [39] [40]. Barriers to enrollment included accessibility relating to weather and travel and appropriate leader/teacher [40]. Perceived health improvement, time of day, opportunities for social engagement/networking, and program pairing were promoters to enrollment among ethnically diverse community dwelling elderly [40]. 
Program pairing refers to variable such as convenient location, time, partnerships with neighborhood organizations, in short the need for easier pathways for activity accessibility [40]. Practicing Tai Chi in a large exercise room with friends can be an opportunity for networking and social engagement. Exercise interventions using group format have resulted in significant gains in exercise motivation, perceived support and information, program satisfaction, and decreased feelings of isolation and loneliness. In our program, this motivator was not utilized fully due to the center's space challenge from building renovation.

These barriers and promoters were reported aggregately instead of separately for ethnicities that are culturally connected to Tai Chi (being East Asian) vs. not connected to Tai Chi. Adaptations addressing Arabic-specific cultural beliefs need to be better understood when translating a Tai Chi fall prevention intervention program to the Arabic community. Arab-Americans had minimal knowledge of Tai Chi, tended to delay access to medical services, and had poor adherence to chronic illness self-care activities comparing with English-speaking Caucasians. They had higher requirement for an interpreter in accessing healthcare [41], looking for someone who not only has the language ability but also share the same origin, religion, dialect, gender and political views with themselves [41]. Although racial stereotyping can be problematic [42], it is a common agreement that disparities are evident among older Arab migrants [42]. A model of clinical and community practice integration [10] suggests that collaborative effort involving multiple providers across different professions in clinical and public health practice will facilitate the transition between clinical practice and community-based interventions. In this model, healthcare providers (e.g., physicians, nurse practitioners) perform a risk assessment and refer patients to a "health coach," who is responsible for identifying and facilitating an appropriate fall-prevention class referral. Community providers (e.g., community centers) are then responsible for enrolling and instructing clients and collecting participation information, which is passed back to the clinician through the health coach to monitor progress. Variations in individual, cultural, and environmental factors need to be recognized to allow implementers to maximize local collaborations of physical activity promotion for the greatest community impact. Our collaboration with the community center could be further developed to address all the system's need.

Cultural sensitivity and competency are terms frequently used in providing nursing care in practice. In fact, cultural sensitivity is equally important when conducting intervention programs with any ethnic minority group since lack of knowledge about participant's culture can be disastrous. Below are specific guidelines to conducting intervention-based health promotion programs with Arab-Americans based on our experience:

1) Partner with a community organization that potential participants currently use and able to transport to unless you plan to provide transportation. Training at least one bilingual staff member (preferably two) from this organization will help improve recruitment and retention of participants. Work with the 
community organization to come up with a timeline or an action plan that meets everyone's needs as much as possible. Be clear about each partners' roles and responsibilities as well as expectations, needs and follow through on commitments.

2) Be flexible when developing the program protocol as you must adapt it to meet the cultural and religious needs of the Arab American population. Schedule more time than usual when explaining your intervention program as ArabAmericans is not used to prevention programs in their home countries.

3) Use direct recruitment by talking to you potential participants, as ArabAmericans are not used to written materials handed to them, by health care providers, in their home countries. They need the personal contact to develop a trusting relationship before they would show interest in an intervention program. In addition, culturally tailored recruitment flyers, may be more helpful than translated general health educational brochures. More research is needed to better understand the linguistic and cultural adaptations of recruitment materials.

4) Develop a trust relationship between potential participants and the team. An Arab American may fear disclosing personal information especially if he or she reside illegally in the United States. Developing a trust relationship is crucial before collecting any data.

5) Translate, translate, and translate. Translate any verbal and written communication with participants to assure full understanding of your intervention program. Using an interpreter whom they trust, and possibly know, is crucial.

6) Correct any misconceptions and misunderstandings about the intervention to improve community acceptance. Misconceptions about physical activity, including that it must be strenuous or uncomfortable to benefit health and a lack of understanding related to how regular, moderate physical activity can benefit health are also problems. You can use your research as an opportunity to conduct mini educational session(s) in the community. This will also help you develop a trust relationship with the community.

7) Include a family member if needed. Family is very important in the Arab-American Culture. It may be important to some participants to include family members in the discussion about participating in the study before deciding to participate in the program.

\section{Conclusion}

Falls are a frequent and costly cause of injuries and functional decline in the elderly. Tai Chi is an evidence-based exercise program endorsed by public health authorities to improve balance and prevent falls in older adults. Many senior centers have introduced Tai Chi programs to increase mobility and decrease the risk of falls. However, the practice has yet to be widely disseminated to ethnic minorities who are not culturally related to Tai Chi. The opportunity that nurse clinicians and researchers to work with community centers in challenging areas involving culturally and ethnically unique older Arab Americans has not been 
sufficiently explored. More efforts are needed to translate and disseminate Tai Chi program to older Arab Americans in community settings. Understanding and paying adequate attention to these challenges that we have shared will enable other nurses to develop similar program, thereby providing support to facilitate the movement of culturally-sensitive community-based health prevention and eventually better access to resources, the implementation of best practices, improved health status in communities, and reduced health care costs.

\section{Acknowledgements}

This program is funded by the Blue Cross Blue Shield of Michigan Foundation.

\section{Conflicts of Interest}

The authors declare that there is no conflict of interest.

\section{References}

[1] Arab American Institute Who Are Arab Americans? http://www.aaiusa.org/who-are-arab-americans

[2] Kridli, S.A. (2002) Health Beliefs and Practices among Arab Women. MCN The American Journal of Maternal Child Nursing, 27, 178-182. https://doi.org/10.1097/00005721-200205000-00010

[3] El-Sayed, A.M., Tracy, M., Scarborough, P. and Galea, S. (2011) Ethnic Inequalities in Mortality: The Case of Arab-Americans. PLoS One, 6, e29185. https://doi.org/10.1371/journal.pone.0029185

[4] Siracuse, J.J., Odell, D.D., Gondek, S.P., Odom, S.R., Kasper, E.M., Hauser, C.J. and Moorman, D.W. (2012) Health Care and Socioeconomic Impact of Falls in the Elderly. The American Journal of Surgery, 203, 335-338; discussion 338. https://doi.org/10.1016/j.amjsurg.2011.09.018

[5] Friedman, S.M., Munoz, B., West, S.K., Rubin, G.S. and Fried, L.P. (2002) Falls and Fear of Falling: Which Comes First? A Longitudinal Prediction Model Suggests Strategies for Primary and Secondary Prevention. Journal of the American Geriatrics Society, 50, 1329-1335. https://doi.org/10.1046/j.1532-5415.2002.50352.x

[6] Dow, B., Meyer, C., Moore, K.J. and Hill, K.D. (2013) The Impact of Care Recipient Falls on Caregivers. Australian Health Review, 37, 152-157. https://doi.org/10.1071/AH12168

[7] Faes, M.C., Reelick, M.F., Joosten-Weyn Banningh, L.W., Gier, M., Esselink, R.A. and Olde Rikkert, M.G. (2010) Qualitative Study on the Impact of Falling in Frail Older Persons and Family Caregivers: Foundations for an Intervention to Prevent Falls. Aging \& Mental Health, 14, 834-842.

https://doi.org/10.1080/13607861003781825

[8] Yao, L., Foley, K.T., Kolanowski, A.M. and Smith, B.A. (2014) Proto Tai Chi: In Search of a Promising Group Exercise for the Frail Elderly. Geriatric Nursing, 35, S21-S26. https://doi.org/10.1016/j.gerinurse.2014.02.021

[9] Li, F., Harmer, P. and Fitzgerald, K. (2016) Implementing an Evidence-Based Fall Prevention Intervention in Community Senior Centers. American Journal of Public Health, 106, 2026-2031. https://doi.org/10.2105/AJPH.2016.303386

[10] Li, F., Eckstrom, E., Harmer, P., Fitzgerald, K., Voit, J. and Cameron, K.A. (2016) Exercise and Fall Prevention: Narrowing the Research-to-Practice Gap and En- 
hancing Integration of Clinical and Community Practice. Journal of the American Geriatrics Society, 64, 425-431. https://doi.org/10.1111/jgs.13925

[11] Yardley, L. and Smith, H. (2002) A Prospective Study of the Relationship between Feared Consequences of Falling and Avoidance of Activity in Community-Living Older People. Gerontologist, 42, 17-23. https://doi.org/10.1093/geront/42.1.17

[12] Panel on Prevention of Falls in Older Persons, American Geriatrics Society and British Geriatrics Society (2011) Summary of the Updated American Geriatrics Society/British Geriatrics Society Clinical Practice Guideline for Prevention of Falls in Older Persons. Journal of the American Geriatrics Society, 59, 148-157. https://doi.org/10.1111/j.1532-5415.2010.03234.x

[13] Yao, L., Giordani, B.J., Algase, D.L., You, M. and Alexander, N.B. (2013) Fall Risk-Relevant Functional Mobility Outcomes in Dementia Following Dyadic Tai Chi Exercise. Western Journal of Nursing Research, 35, 281-296. https://doi.org/10.1177/0193945912443319

[14] Hauer, K.A., Kempen, G.I., Schwenk, M., Yardley, L., Beyer, N., Todd, C., Oster, P. and Zijlstra, G.A. (2010) Validity and Sensitivity to Change of the Falls Efficacy Scales International to Assess Fear of Falling in Older Adults with and without Cognitive Impairment. Gerontology. https://doi.org/10.1159/000320054

[15] Delbaere, K., Close, J.C., Mikolaizak, A.S., Sachdev, P.S., Brodaty, H. and Lord, S.R. (2010) The Falls Efficacy Scale International (FES-I). A Comprehensive Longitudinal Validation Study. Age Ageing, 39, 210-216. https://doi.org/10.1093/ageing/afp225

[16] Halaweh, H., Svantesson, U., Rosberg, S. and Willen, C. (2016) Cross-Cultural Adaptation, Validity and Reliability of the Arabic Version of the Falls Efficacy Scale-International (FES-I). Medical Principles and Practice: International Journal of the Kuwait University, Health Science Centre, 25, 1-7.

[17] Shumway-Cook, A., Brauer, S. and Woollacott, M. (2000) Predicting the Probability for Falls in Community-Dwelling Older Adults Using the Timed up \& Go Test. Physical Therapy, 80, 896-903.

[18] Podsiadlo, D. and Richardson, S. (1991) The Timed "Up \& Go": A Test of Basic Functional Mobility for Frail Elderly Persons. Journal of the American Geriatrics Society, 39, 142-148. https://doi.org/10.1111/j.1532-5415.1991.tb01616.x

[19] Alexander, N.B. and Goldberg, A. (2005) Gait Disorders: Search for Multiple Causes. Cleveland Clinic Journal of Medicine, 72, 589-590.

[20] Vellas, B.J., Wayne, S.J., Romero, L., Baumgartner, R.N., Rubenstein, L.Z. and Garry, P.J. (1997) One-Leg Balance Is an Important Predictor of Injurious Falls in Older Persons. Journal of the American Geriatrics Society, 45, 735-738. https://doi.org/10.1111/j.1532-5415.1997.tb01479.x

[21] Van Iersel, M.B., Munneke, M., Esselink, R.A., Benraad, C.E. and Olde Rikkert, M.G. (2008) Gait Velocity and the Timed-Up-and-Go Test Were Sensitive to Changes in Mobility in Frail Elderly Patients. Journal of Clinical Epidemiology, 61, 186-191. https://doi.org/10.1016/j.jclinepi.2007.04.016

[22] Van Iersel, M.B., Benraad, C.E. and Rikkert, M.G. (2007) Validity and Reliability of Quantitative Gait Analysis in Geriatric Patients with and without Dementia. Journal of the American Geriatrics Society, 55, 632-634.

https://doi.org/10.1111/j.1532-5415.2007.01130.x

[23] Lamb, S.E., Jorstad-Stein, E.C., Hauer, K. and Becker, C. (2005) Development of a Common Outcome Data Set for Fall Injury Prevention Trials: The Prevention of Falls Network Europe Consensus. Journal of the American Geriatrics Society, 53, 1618-1622. https://doi.org/10.1111/j.1532-5415.2005.53455.x 
[24] CDC. Preventing Falls: How to Develop Community-Based Fall Prevention Programs for Older Adults.

[25] Ramanadhan, S., Crisostomo, J., Alexander-Molloy, J., Gandelman, E., Grullon, M., Lora, V., Reeves, C., Savage, C., Viswanath, K. and C-PAC, P.M. (2012) Perceptions of Evidence-Based Programs among Community-Based Organizations Tackling Health Disparities: A Qualitative Study. Health Education Research, 27, 717-728. https://doi.org/10.1093/her/cyr088

[26] MacEntee, M.I., Wyatt, C., Kiyak, H.A., Hujoel, P.P., Persson, R.E., Persson, G.R. and Powell, L.V. (2002) Response to Direct and Indirect Recruitment for a Randomised Dental Clinical Trial in a Multicultural Population of Elders. Community Dentistry and Oral Epidemiology, 30, 377-381. https://doi.org/10.1034/j.1600-0528.2002.00003.x

[27] George, S., Duran, N. and Norris, K. (2014) A Systematic Review of Barriers and Facilitators to Minority Research Participation among African Americans, Latinos, Asian Americans, and Pacific Islanders. American Journal of Public Health, 104, e16-e31. https://doi.org/10.2105/AJPH.2013.301706

[28] Yancey, A.K., Ortega, A.N. and Kumanyika, S.K. (2006) Effective Recruitment and Retention of Minority Research Participants. Annual Review of Public Health, 27, 1-28. https://doi.org/10.1146/annurev.publhealth.27.021405.102113

[29] Sickinger, K., Walker, W.C., Agyemang, A.A., Cifu, D.X., Lewis, T.L. and Carne, W. (2018) Recruiting for a Multicentre DoD and VA Longitudinal Study: Lessons Learned. Brain Injury, 32, 1218-1225. https://doi.org/10.1080/02699052.2018.1492740

[30] Brawley, L.R., Rejeski, W.J. and King, A.C. (2003) Promoting Physical Activity for Older Adults: The Challenges for Changing Behavior. American Journal of Preventive Medicine, 25, 172-183. https://doi.org/10.1016/S0749-3797(03)00182-X

[31] Alzubaidi, H., Mc Namara, K., Browning, C. and Marriott, J. (2015) Barriers and Enablers to Healthcare Access and Use among Arabic-Speaking and Caucasian English-Speaking Patients with Type 2 Diabetes Mellitus: A Qualitative Comparative Study. BMJ Open, 5, e008687. https://doi.org/10.1136/bmjopen-2015-008687

[32] Alzubaidi, H., Mc Narmara, K., Kilmartin, G.M., Kilmartin, J.F. and Marriott, J. (2015) The Relationships between Illness and Treatment Perceptions with Adherence to Diabetes Self-Care: A Comparison between Arabic-Speaking Migrants and Caucasian English-Speaking Patients. Diabetes Research and Clinical Practice, 110, 208-217. https://doi.org/10.1016/j.diabres.2015.08.006

[33] Resnicow, K., Baranowski, T., Ahluwalia, J.S. and Braithwaite, R.L. (1999) Cultural Sensitivity in Public Health: Defined and Demystified. Ethnicity \& Disease, 9, 10-21.

[34] Resnicow, K., Braithwaite, R.L., Dilorio, C. and Glanz, K. (2002) Applying Theory to Culturally Diverse and Unique Populations. In: Glanz, K., Lewis, F.M. and Rimer, B.K., Eds., Health Behavior and Health Education: Theory, Research, and Practice, 3rd Edition, Jossey-Bass Publishers, San Francisco, 485-509.

[35] Li, F. and Harmer, P. (2015) Economic Evaluation of a Tai Ji Quan Intervention to Reduce Falls in People with Parkinson Disease, Oregon, 2008-2011. Preventing Chronic Disease, 12, E120. https://doi.org/10.5888/pcd12.140413

[36] Manson, J., Ritvo, P., Ardern, C., Weir, P., Baker, J., Jamnik, V. and Tamim, H. (2013) Tai Chi's Effects on Health-Related Fitness of Low-Income Older Adults. Canadian Journal on Aging, 32, 270-277. https://doi.org/10.1017/S0714980813000305

[37] Manson, J., Rotondi, M., Jamnik, V., Ardern, C. and Tamim, H. (2013) Effect of Tai 
Chi on Musculoskeletal Health-Related Fitness and Self-Reported Physical Health Changes in Low Income, Multiple Ethnicity Mid to Older Adults. BMC Geriatrics, 13, 114. https://doi.org/10.1186/1471-2318-13-114

[38] Woods, N.F., Cochrane, B.B., LaCroix, A.Z., Seguin, R.A., Zaslavsky, O., Liu, J., Beasley, J.M., Brunner, R.L., Espeland, M.A., Goveas, J.S., et al. (2012) Toward a Positive Aging Phenotype for Older Women: Observations from the Women's Health Initiative. The Journals of Gerontology. Series A, Biological Sciences and Medical Sciences, 67, 1191-1196. https://doi.org/10.1093/gerona/gls117

[39] Dogra, S., Al-Sahab, B., Manson, J. and Tamim, H. (2015) Aging Expectations Are Associated with Physical Activity and Health among Older Adults of Low Socioeconomic Status. Journal of Aging and Physical Activity, 23, 180-186. https://doi.org/10.1123/japa.2012-0337

[40] Manson, J.D., Tamim, H. and Baker, J. (2017) Barriers and Promoters for Enrollment to a Community-Based Tai Chi Program for Older, Low-Income, and Ethnically Diverse Adults. Journal of Applied Gerontology. The Official Journal of the Southern Gerontological Society, 36, 592-609.

[41] Hadziabdic, E. and Hjelm, K. (2014) Arabic-Speaking Migrants' Experiences of the Use of Interpreters in Healthcare: A Qualitative Explorative Study. International Journal for Equity in Health, 13, 49. https://doi.org/10.1186/1475-9276-13-49

[42] Al Abed, N.A., Davidson, P.M. and Hickman, L.D. (2014) Healthcare Needs of Older Arab Migrants: A Systematic Review. Journal of Clinical Nursing, 23, 1770-1784. https://doi.org/10.1111/jocn.12476 